\title{
Numerical Range on Weighted Hardy Spaces as Semi Inner Product Spaces
}

\author{
Mohammad Taghi Heydari
}

\begin{abstract}
The semi-inner product, in the sense of Lumer, on weighted Hardy space which generate the norm is unique. Also we will discuss some properties of the numerical range of bounded linear operators on weighted Hardy spaces.
\end{abstract}

\section{Introduction}

Let $T$ be a (bounded linear) operator on a complex Hilbert space $\mathcal{H}$. The numerical range of $T$ is the set

$$
W(T):=\{\langle T x, x\rangle: x \in \mathcal{H},\|x\|=1\}
$$

in the complex plane, where $\langle.,$.$\rangle denotes the inner product in \mathcal{H}$. In other words, $W(T)$ is the image of the unit sphere $\{x \in \mathcal{H}:\|x\|=1\}$ of $\mathcal{H}$ under the (bounded) quadratic form $x \mapsto\langle T x, x\rangle$.

Some properties of the numerical range follow easily from the definition. For one thing, the numerical range is unchanged under the unitary equivalence of operators: $W(T)=W\left(U^{*} T U\right)$ for any unitary $U$. It also behaves nicely under the operation of taking the adjoint of an operator: $W\left(T^{*}\right)=\{\bar{z}: z \in$ $W(T)\}$. One of the most fundamental properties of the numerical range is its convexity, stated by the famous Toeplitz-Hausdorff Theorem. It is known that

Key Words: weighted Hardy space, semi-inner product, numerical range.

2010 Mathematics Subject Classification: Primary 30H10; Secondary 47B37, 47A12.

Received: 1.03 .2016

Accepted: 28.03.2016 
$W(T)$ is a connected set and its closure contains the spectrum of $T$. Also in the finite dimensional case, $W(T)$ is compact.

By the contrast to the long history of the Hilbert space numerical range, the birth of the general theory was long delayed and its growth has been spectacular. No concept of numerical range appropriate to general normed linear spaces appeared until 1961 and 1962, when distinct, though related, concepts were introduced independently by Bauer [2] and Lumer [13].

Let $X$ be a complex vector space. The mapping $[\cdot, \cdot]: X \times X \rightarrow \mathbb{C}$ is called a semi-inner product, in the sense of Lumer, if the following properties are satisfied:

(i) $[x+y, z]=[x, z]+[y, z]$ for all $x, y, z \in X$;

(ii) $[\lambda x, y]=\lambda[x, y]$ for all $x, y \in X$ and $\lambda \in \mathbb{C}$;

(iii) $[x, x] \geq 0$ for all $x \in X$;

(iv) $|[x, y]|^{2} \leq[x, x][y, y]$ for all $x, y \in X$ and $\lambda \in \mathbb{C}$.

Lumer [13] showed that a semi-inner product space is a normed linear space with the norm $[x, x]^{\frac{1}{2}}$. On the other hand every normed linear space $(X,\|\cdot\|)$ has at least one semi-inner product $[.,$.$] such that$

$$
[x, x]=\|x\|^{2} \quad(x \in X) .
$$

If a semi-inner product satisfying (1.2), the definition of numerical range used for Hilbert spaces at once generalizes to give the definition of the numerical range $W(T)$ for a linear operator on $X$,

$$
W(T)=\{[T x, x]:\|x\|=1\} .
$$

On the face of this definition has the serious defect that it is not an invariant of the normed space $(X,\|\|$.$) . There are many semi-inner-product(infinite) on$ $X$ satisfying (1.2) except when the unit ball of $X$ is smooth(i.e., for all $x$, with $\|x\|=1$, there is a unique $x^{*}$ in dual space $X, X^{*}$, such that $\left\|x^{*}\right\|=1$ and $\left.x^{*}(x)=1\right)$.

Lumer proved that, $\overline{c o} W(T)$, the closed convex hull of $W(T)$, is independent of the choice of semi-inner product satisfying (1.2). In fact, he showed that $\overline{c o} W(T)$ depends only on the norms of the operators in two dimensional linear subspace spanned by $I$, the identity operator, and $T$.

In this paper, we establish a fundamental proposition, which says that the unit ball of weighted Hardy spaces is smooth. By this proposition we show 
that there is one and only one semi-inner product on weighted Hardy spaces in Lumer's sense which satisfies (1.2). In addition, some properties of the numerical range of bounded linear operators on weighted Hardy spaces are discussed.

\section{2 weighted Hardy space}

In this section, we recall the definition of the weighted Hardy spaces, which will be used in the sequel.

Let $1<p<\infty$ and $\{\beta(n)\}_{n}$ be a sequence of positive numbers with $\beta(0)=1$. The weighted Hardy space, which is denoted by $H^{p}(\beta)$, is the set of all formal power series $f(z)=\sum_{n=0}^{\infty} \hat{f}(n) z^{n}$ with

$$
\|f\|^{p}=\|f\|_{H^{p}(\beta)}^{p}=\sum_{n=0}^{\infty}|\hat{f}(n)|^{p} \beta(n)^{p}<\infty .
$$

Let $\mu(K)=\sum_{n \in K} \beta(n)^{p}$, for $K \subseteq \mathbb{N} \cup\{0\}$. Then $\mu$ is a $\sigma$-finite measure and $H^{p}(\beta)=L^{p}(\mu)$. So the space $H^{p}(\beta)$ is a reflexive Banach space with the norm $\|\cdot\|_{H^{p}(\beta)}$, and the dual of $H^{p}(\beta)$ is $H^{q}\left(\beta^{\frac{p}{q}}\right)$, where $1 / p+1 / q=1$ and $\beta^{p / q}=\left\{\beta(n)^{p / q}\right\}$ (see, [14]).

In the case $p=2$, the weighted Hardy spaces with $\beta(n)=1, \beta(n)=$ $(n+1)^{\frac{-1}{2}}$ and $\beta(n)=(n+1)^{\frac{1}{2}}$ are classical Hardy space, Bergman space and the Dirichlet space, respectively (see [6], [8], [18] for more about them).

The space $H^{2}(\beta)$ becomes to a Hilbert space with inner product

$$
\langle f, g\rangle=\sum_{n=0}^{\infty} a_{n} b_{n} \beta(n)^{2}
$$

where $f(z)=\sum a_{n} z^{n}$ and $g(z)=\sum b_{n} z^{n}$ are the elements of $H^{2}(\beta)$ (see, [15]).

The notation $\langle f, g\rangle$ is to stand for $g(f)$ where $f \in H^{p}(\beta)$ and $g \in\left(H^{p}(\beta)\right)^{*}$. Note that (see, [17]):

$$
\langle f, g\rangle=\sum_{n=0}^{\infty} \hat{f}(n) \overline{\hat{g}(n)} \beta(n)^{p} .
$$

For $f \in H^{p}(\beta)$ and $g \in H^{q}\left(\beta^{\frac{p}{q}}\right)$, with $f(z)=\sum a_{n} z^{n}$ and $g(z)=\sum b_{n} z^{n}$, we define $f^{*}$ and ${ }^{*} g$ by $f^{*}=\sum\left|a_{n}\right|^{p-1} \operatorname{sgn}\left(a_{n}\right) z^{n}$ and ${ }^{*} g=\sum\left|b_{n}\right|^{q-1} \operatorname{sgn}\left(b_{n}\right) z^{n}$ 
respectively, where $\operatorname{sgn}(0)=0$ and $\operatorname{sgn}(w)=\frac{w}{|w|}$ for a nonzero complex number $w$. Clearly

$$
\left\|f^{*}\right\|_{q}^{q}=\left\|f^{*}\right\|_{H^{q}\left(\beta^{\left.\frac{p}{q}\right)}\right.}^{q}=\sum_{n=0}^{\infty}|\hat{f}(n)|^{p} \beta(n)^{p}=\|f\|_{p}^{p}<\infty,
$$

and

$$
\left\|^{*} g\right\|_{p}^{p}=\sum_{n=0}^{\infty}|\hat{g}(n)|^{q} \beta(n)^{p}=\|g\|_{q}^{q}<\infty .
$$

Thus $f^{*} \in H^{q}\left(\beta^{\frac{p}{q}}\right)$ and ${ }^{*} g \in H^{p}(\beta)$. Obviously, one can see that ${ }^{*}\left(f^{*}\right)=f$ for all $f \in H^{p}(\beta)$ and $\left({ }^{*} g\right)^{*}=g$ for all $g$ in $\left(H^{p}(\beta)\right)^{*}$. By a simple computation we also have the following consequences:

a) If $\alpha \geq 0$ and $f \in H^{p}(\beta)$ then $(\alpha f)^{*}=\alpha^{p-1} f^{*}$

b) If $f \in H^{p}(\beta),\left\langle f, f^{*}\right\rangle=\|f\|_{p}^{p}$

Proposition 2.1. The unit ball of $H^{p}(\beta)$ is smooth.

Proof. Suppose $f \in H^{p}(\beta), g \in\left(H^{p}(\beta)\right)^{*},\|f\|=\|g\|=1$ and $\langle f, g\rangle=1$. Then

$$
1=\langle f, g\rangle \leq\|f\|\|g\|=1 .
$$

Therefore equality occurs in Holder inequality and there are complex numbers $\alpha$ and $\eta$ (independent of $\mathrm{n}$ ) such that $|\hat{f}(n)|^{p} \beta(n)^{p}=\alpha|\hat{g}(n)|^{q} \beta(n)^{p}$ and $\arg (\hat{f}(n) \overline{\hat{g}(n)})=\eta$ (see [11]). Hence $|\hat{f}(n)|^{p}=\alpha|\hat{g}(n)|^{q}$. But

$$
1=\|f\|_{p}^{p}=\sum|\hat{f}(n)|^{p} \beta(n)^{p}=\alpha \sum|\hat{g}(n)|^{q} \beta(n)^{p}=\alpha,
$$

and hence $|\hat{f}(n)|^{p}=|\hat{g}(n)|^{q}$. On the other hand

$$
\begin{aligned}
1 & =\sum \hat{f}(n) \overline{\hat{g}(n)} \beta(n)^{p} \\
& =\sum|\hat{f}(n)||\hat{g}(n)| e^{i \arg (\hat{f}(n) \overline{\hat{g}(n)})} \beta(n)^{p} \\
& =e^{i \eta} \sum|\hat{f}(n) \| \hat{f}(n)|^{p / q} \beta(n)^{p}=e^{i \eta} .
\end{aligned}
$$

Therefore $e^{\operatorname{iarg}(\hat{f}(n) \overline{\hat{g}(n)})}=1$, or equivalently $e^{\operatorname{iarg}(\hat{f}(n))}=e^{\operatorname{iarg}(\hat{g}(n))}$. This implies

$$
\hat{g}(n)=|\hat{f}(n)|^{p / q} e^{i a r g(\hat{f}(n))},
$$

or

$$
g=f^{*} .
$$

Hence the unit ball of $H^{p}(\beta)$ is smooth. 
Define a semi-inner product on $H^{p}(\beta)$ by

$$
[g, f]:=\left\langle g, F_{f}\right\rangle,
$$

where $f, g \in H^{p}(\beta)$ and $F_{f}:=\|f\|^{2-p} f^{*}$. Obviously, we have $[f, f]=\|f\|_{p}^{2}$. Thus, by Proposition 2.1, it is the only semi-inner product on weighted Hardy space in Lumer's sense on $H^{p}(\beta)$ which satisfies (1.2).

\section{3 results and discussion}

In this section we use the proposition 2.1 as a way to guide the discussion. But first, we recall some other basic properties of weighted Hardy spaces.

Suppose $f \in H^{p}(\beta)$, our definition of $f^{*}$ implies:

- ${ }^{*}\left(f^{*}\right)=f$ for all $f \in H^{p}(\beta)$ and $\left({ }^{*} g\right)^{*}=g$ for all $g$ in $\left(H^{p}(\beta)\right)^{*}$.

- $f \rightarrow f^{*}$ is a bijection from $H^{p}(\beta)$ onto $\left(H^{p}(\beta)\right)^{*}$ that is isometry.

Also it has the additional nice properties, i.e.,

- The fundamental proposition 2.1.

- There is no ambiguity in the numerical range with respect to our definition. Indeed, in terms of a semi-inner-product satisfying (1.2), the definition of usual numerical range for Hilbert space operator at once generalizes to give the definition of the numerical range $W(T)$ for a linear operator on $X$,

$$
W(T)=\{[T x, x]:\|x\|=1\} .
$$

In most cases, there are infinitely many semi-inner products on $X$ satisfying (1.2). But, for bounded linear operator $T$ on weighted Hardy space $H^{p}(\beta)$ the spatial numerical range of $T, V(T)$, coincides with numerical range $W(T)$, Indeed,

$$
\begin{aligned}
W(T) & =V(T) \\
& :=\left\{\left\langle T x, x^{*}\right\rangle: x \in X, x^{*} \in X^{*},\|x\|=\left\|x^{*}\right\|=\left\langle x, x^{*}\right\rangle=1\right\} .
\end{aligned}
$$

where $W(T)$ is the numerical range of $T$ respect to the semi inner product defined by (2.1). Therefore

$$
V(T)=W(T)=\left\{\left\langle T f, f^{*}\right\rangle: f \in H^{p}(\beta),\|f\|=1\right\} .
$$


In the following, we discuss some properties of the numerical range of a bounded linear operator $T$ on $H^{p}(\beta)$ and show that it is need not be convex, even if $T$ is compact.

Recall that the usual notation $\sigma(T), \sigma_{p}(T)$ and $\sigma_{a p}(T)$ for the spectrum, point spectrum and approximate point spectrum of $T$ respectively.

Theorem 3.1. Let $T$ be a bounded linear operator on $H^{p}(\beta)$. Then

(i) $W(T)=W\left(T^{*}\right)$;

(ii) $W(T)$ need not be convex;

(iii) $\sigma_{p}(T) \subseteq W(T)$;

(iv) $\sigma(T) \subseteq \overline{W(T)}$, the closure of $W(T)$.

Proof. (i): By [ [4],Corollary 9.6] we have $W(T) \subseteq W\left(T^{*}\right)$. For the other hand since $H^{p}(\beta)$ is reflexive Banach space and $\left.T^{* *}\right|_{H^{p}(\beta)}=T$, we have

$$
W\left(T^{*}\right) \subseteq W\left(T^{* *}\right)=W(T) .
$$

(ii): Let $\beta(1)=1$ and $T$ be the linear operator on $H^{p}(\beta)$ defined by

$$
(\widehat{T f})(n)=\left\{\begin{array}{cc}
i \hat{f}(0)+\hat{f}(1) & n=0 \\
-(\hat{f}(0)+i \hat{f}(1)) & n=1 \\
0 & n>1
\end{array}\right.
$$

Therefore

$$
\begin{aligned}
W(T) & =\left\{\left\langle T f, f^{*}\right\rangle:\|f\|=1, f \in H^{p}(\beta)\right\} \\
& =\left\{(\widehat{T f})(0)|\hat{f}(0)|^{\frac{p}{q}} e^{-i \theta_{0}}+(\widehat{T f})(1)|\hat{f}(1)|^{\frac{p}{q}} e^{-i \theta_{1}}:\|f\|=1, f \in H^{p}(\beta)\right\},
\end{aligned}
$$

where $\theta_{0}=\arg (\hat{f}(0))$ and $\theta_{1}=\arg (\hat{f}(1))$. By writing

$$
|\hat{f}(0)|=r,|\hat{f}(1)|=s, \theta=\theta_{1}-\theta_{0}
$$

, we have

$W(T)=\left\{r s\left(r^{p-2}-s^{p-2}\right) \cos \theta+i\left[r^{p}-s^{p}+r s\left(r^{p+2}+s^{p+2}\right) \sin \theta\right]: r^{p}+s^{p} \leq 1\right\}$.

Now, let

$$
\alpha=\sup \{\operatorname{Re} z: z \in W(T)\}=\sup \left\{r s\left(r^{p-2}-s^{p-2}\right): r^{p}+s^{p} \leq 1\right\},
$$

and

$$
\begin{aligned}
\beta= & \sup \{W(T) \cap \mathbb{R}\} \\
= & \sup \left\{\cos \theta \cdot r s\left(r^{p-2}-s^{p-2}\right):\right. \\
& \left.r^{p}+s^{p} \leq 1 \text { and } r^{p}-s^{p}+r s\left(r^{p+2}+s^{p+2}\right) \sin \theta=0\right\}
\end{aligned}
$$


We have $\alpha>\beta$ unless $p=2$. If

$$
z=r s\left(r^{p-2}-s^{p-2}\right) \cos \theta+i\left[r^{p}-s^{p}+r s\left(r^{p+2}+s^{p+2}\right) \sin \theta\right] \in W(T)
$$

then the conjugate of $z$ is

$\bar{z}=\operatorname{sr}\left(s^{p-2}-r^{p-2}\right) \cos (\pi+\theta)+i\left[s^{p}-r^{p}+\operatorname{sr}\left(s^{p+2}+r^{p+2}\right) \sin (\pi+\theta)\right] \in W(T)$

and $W(T)$ is symmetry with respect to real axis. Thus $\alpha$ attained at points above and below the real axis, and we obtain that $W(T)$ is not convex unless $p=2$ (see also [1]).

(iii): Let $\lambda \in \sigma_{p}(T)$. Then there exists a non zero function $f \in H^{p}(\beta)$ such that $T f=\lambda f$. Put $g=\frac{f}{\|f\|_{p}}$. Then, by property of .* and $\langle.,$.$\rangle in weighted$ Hardy space, we have

$$
\begin{aligned}
\left\langle T g, g^{*}\right\rangle & =\left\langle\frac{\lambda}{\|f\|_{p}} f, \frac{1}{\|f\|_{p}^{p-1}} f^{*}\right\rangle \\
& =\frac{\lambda}{\|f\|_{p}^{p}}\left\langle f, f^{*}\right\rangle \\
& =\frac{\lambda}{\|f\|_{p}^{p}}\|f\|_{p}^{p} \\
& =\lambda .
\end{aligned}
$$

Hence $\lambda \in W(T)$.

(iv): This part is proved by Williams in [16] for Banach spaces, but the following proof for weighted Hardy spaces is elementary. Let $\lambda \in \sigma_{a p}(T)$. There exists a sequence $\left\{f_{n}\right\}$ in $H^{p}(\beta)$ such that $\left\|f_{n}\right\|_{p}=1$ and $(T-\lambda) f_{n} \rightarrow 0$ as $n \rightarrow \infty$. Thus

$$
\left\langle T f_{n}, f_{n}^{*}\right\rangle \rightarrow \lambda\left\langle f_{n}, f_{n}^{*}\right\rangle=\lambda\left\|f_{n}\right\|_{p}^{p}=\lambda
$$

as $n \rightarrow \infty$ and $\lambda \in \overline{W(T)}$. Then (iv) hold by (i), (ii) and the identity

$$
\sigma(T)=\sigma_{a p}(T) \bigcup \sigma_{p}\left(T^{*}\right) .
$$

In the following theorem, we prove an interesting result for compact operators acting on $H^{p}(\beta)$. 
Theorem 3.2. If $T$ is a compact operator on $H^{p}(\beta)$, then $\overline{c o} W(T)$ obtained by connecting all points in $W(T)$ to the origin. In fact

$$
\overline{c o} W(T)=\operatorname{co}(\{0\} \bigcup W(T)) .
$$

Proof. Since $T$ is compact on an infinite dimensional Banach space, we have $0 \in \sigma(T)$. By part (iv) of Theorem 3.1 we have $0 \in \overline{W(T)}$ that implies

$$
\operatorname{co}(\{0\} \bigcup W(T)) \subseteq \overline{c o} W(T)
$$

For the other hand, let $\alpha \in \overline{W(T)}$. Then there exists a sequence $\left\{f_{n}\right\} \in H^{p}(\beta)$ with $\left\|f_{n}\right\|_{p}=1$ and $\left\langle T f_{n}, f_{n}^{*}>\rightarrow \alpha\right.$. By reflexivity of $H^{p}(\beta)$ and BanachAlaoglu theorem, there exists a subsequence of $\left\{f_{n}\right\}$, without loss of generality, assume $\left\{f_{n}\right\}$, such that $f_{n} \rightarrow f$ in weak topology for some $f$ in $H^{p}(\beta)$ with $\left\|f_{n}\right\|_{p} \leq 1$ and $f_{n}^{*} \rightarrow f^{*}$ in weak-star topology.

Then

$$
\left\langle T f, f_{n}^{*}-f^{*}\right\rangle \rightarrow 0,
$$

and since $T$ is compact, we have

$$
T f_{n} \rightarrow T f
$$

Also

$$
\begin{aligned}
\left|\left\langle T f_{n}, f_{n}^{*}\right\rangle-\left\langle T f, f^{*}\right\rangle\right| & =\left|\left\langle T f_{n}, f_{n}^{*}\right\rangle-\left\langle T f, f^{*}\right\rangle \pm\left\langle T f, f_{n}^{*}\right\rangle\right| \\
& =\left|\left\langle T f_{n}-f, f_{n}^{*}\right\rangle+\left\langle T f, f_{n}^{*}-f^{*}\right\rangle\right| \\
& \leq \| T\left(f_{n}-f\right)|||| f_{n}^{*}||+\left|\left\langle T f, f_{n}^{*}-f^{*}\right\rangle\right| \rightarrow 0 .
\end{aligned}
$$

Hence, $\left\langle T f_{n}, f_{n}^{*}\right\rangle \rightarrow\left\langle T f, f^{*}\right\rangle$ as $n \rightarrow \infty$ and so indeed $\alpha=\left\langle T f, f^{*}\right\rangle$.

If $\alpha \neq 0$, then $f \neq 0$ and

$$
\alpha=\|f\|_{p}^{p}\left\langle T\left(\frac{f}{\|f\|_{p}}\right),\left(\frac{f}{\|f\|_{p}}\right)^{*}\right\rangle \in\|f\|_{p}^{p} W(T),
$$

thus

$$
\overline{c o} W(T) \subseteq \operatorname{co}(\{0\} \bigcup W(T)),
$$

and so the proof is complete.

Corollary 3.3. Let $T$ be a compact operator on $H^{p}(\beta)$. If $W(T)$ is closed then $0 \in W(T)$. Also, if $0 \in W(T)$ then $c o(W(T))$ is closed. 
Corollary 3.4. If $T$ is a compact operator on $H^{p}(\beta)$ such that $W(T)$ is star shaped respect to zero i.e., $t z \in W(T)$ for $0 \leq t \leq 1$ and $z \in W(T)$. Then $W(T)$ is closed.

Proof. Since $W(T)$ is star shaped respect to zero then we have (see [1])

$$
W(T)=\left\{\left\langle T f, f^{*}\right\rangle:\|f\|_{p} \leq 1\right\} .
$$

Let $\alpha \in \overline{W(T)}$, then $\alpha=\left\langle T f, f^{*}\right\rangle$ for some $f$ in $H^{p}(\beta)$ with $\left\|f_{n}\right\|_{p} \leq 1$ (Theorem 3.2). Thus $\alpha \in W(T)$ and so $W(T)$ is closed.

Remark 3.5. Uniformly convex spaces (or uniformly rotund spaces) are common examples of reflexive Banach spaces. The concept of uniform convexity was first introduced by James A. Clarkson in 1936 (see [5]).

A uniformly convex space is a normed vector space so that, for every $0<\epsilon \leq 2$ there is some $\delta>0$ so that for any two vectors with $\|x\|=1$ and $\|y\|=1$, the condition

$$
\|x-y\| \geq \varepsilon
$$

implies that:

$$
\left\|\frac{x+y}{2}\right\| \leq 1-\delta
$$

Intuitively, the center of a line segment inside the unit ball must lie deep inside the unit ball unless the segment is short. The Milman-Pettis theorem states that every uniformly convex Banach space is reflexive, while the converse is not true.

Let $T$ be a bounded linear operator on $H^{p}(\beta)$. Since $H^{p}(\beta)$ is uniformly convex (See [4]), then we have the following:

- $\partial \sigma(T) \subseteq \overline{W(T)}$

- $\{\lambda: \lambda \in \overline{W(T)},|\lambda|=|| T||\} \subseteq \partial \sigma(T)$

- If $\sup \{|\lambda|: \lambda \in W(T)\}=\|T\|$, then $\sup \{|\lambda|: \lambda \in \sigma(T)\}=\|T\|$.

- Since $H^{p}(\beta)$ is strictly convex (i.e., $f \neq 0, g \neq 0$ and $\|f+g\|_{p}=$ $\|f\|_{p}+\|g\|_{p}$ imply that $f=c g$ for some constant $\left.c>0\right)$. We have that if $\lambda \in W(T)$ and $|\lambda|=\|T\|$, then $\lambda$ is an eigenvalue of $T$. 
Remark 3.6. Let the numerical index of a normed space $X$ be the real number $n(X)$ defined by

$$
n(X)=\inf \{v(T): T \in B(X),\|T\|=1\}
$$

where

$$
v(T)=\sup \{|\lambda|: \lambda \in V(T)\}
$$

is the numerical radius of $T$. Clearly, $v$ is a semi-norm on $B(X)$, and $v(T) \leq$ $\|T\|$ for every $T \in B(X)$. It was shown by Glickfeld [9] (and essentially by Bohnenblust and Karlin [3]) that if $X$ is a complex space, then $e^{-1}|| T|| \leq v(T)$ for every $T \in B(X)$ where $e=\exp 1$, so that for complex spaces, $v$ is always a norm and it is equivalent to the operator norm $\|$.$\| .$

Obviously, $n(X)$ is the greatest constant $k \geq 0$ such that $k\|T\| \leq v(T)$ for every $T \in B(X)$. Note that for any complex Banach space $X, e^{-1} \leq n(X) \leq 1$. The concept of the numerical index was first suggested by Lumer [13]. At that time, it was known that if $X$ is a complex Hilbert space (with $\operatorname{dim} X>1$ ) then $n(X)=\frac{1}{2}$ and if it is real then $n(X)=0$ so that for real spaces, $0 \leq n(X) \leq 1$.

Later, Duncan et al. [4] determined the range of values of the numerical index. More precisely, they proved that

$$
\begin{gathered}
\{n(X): X \text { real Banach }\}=[0,1], \\
\{n(X): X \text { complex Banach space }\}=\left[e^{-1}, 1\right] .
\end{gathered}
$$

As an interesting open problem is to compute the numerical index of $H^{p}(\beta)$. Since $H^{p}(\beta)=L^{p}(\mu)$ for some $\mu$, so the $n\left(H^{p}(\beta)\right)$ is dominated by $n\left(\ell_{p}\right)$. For more details see [7].

\section{References}

[1] A. Abdollahi and M. T. Heydari, Spatial Numerical Range of Operators on Weighted Hardy Spaces, International Journal of Mathematics and Mathematical Sciences. DOI:10.1155/2011/812680

[2] F. L. Bauer, On the Filed of Valuse Subordinates to a Norm, Numer. Math. 4 (1961) 103-111.

[3] H.F. Bohnenblust, S. Karlin, Geometrical properties of the unit sphere in Banach algebra, Ann. Math. 62 (1955) 217-229.

[4] F. F. Bonsall and J. Duncan, Numerical Ranges of Operators on Normed Spaces and of Elements of Normed Algebras, London Math. Soc. Lecture Notes Series 2, Cambridge, 1971. 
[5] J. A. Clarkson, Uniformly Convex Spaces, Trans. Amer. Math. Soc. 40 (1936): 396-414

[6] C. C. Cowen and B. D. MacCluer, Composition Operators on Spaces of Analytic Functions, CRC Press, 1995

[7] Elmouloudi Ed-dari, On the numerical index of Banach spaces, Linear Algebra and its Applications 403 (2005) 86-96.

[8] E. A. Gallardo-Gutierrez and A. Montes-Rodriguez, The role of the spectrum in the cyclic behavior of composition operators, Mem. Amer. Math. Soc. 167 (2004), no. 791, x+81 pp.

[9] B.W. Glickfeld, On an inequality of Banach algebra geometry and semiinner product space theory, Illinois J. Math. 14 (1970) 76-81.

[10] Gustafon, K. E., K. M. Rao.: The numerical range, the field of values of linear operators and matrices. Springer. New York. 1997.

[11] L. P. Kuptsov, Hölder inequality, in Hazewinkel, Michiel, Encyclopaedia of Mathematics, Kluwer Academic Publishers, 2001, ISBN 9781556080104 .

[12] K. Jahedi and B. Yousefi, Numerical Range of Operators Acting on Banach Spaces, Czechoslovak Mathematical Journal, Vol. 62 (2012), No. 2, 495-503.

[13] G. Lumer, Semi-inner-product spaces, Trans. Amer. Math. Soc. 1001961 29-43.

[14] K. Seddighi, K. Hedayatiyan and B. Yousefi, Operators acting on certain Banach spaces of analytic functions, Internat. J. Math. Math. Sci. 18 (1995), no. 1, 107-110.

[15] A. L. Shields, Weighted shift operators and analytic function theory, Topics in operator theory, pp. 49-128. Math. Surveys, No. 13, Amer. Math. Soc., Providence, R.I., 1974.

[16] J. P. Williams, Spectra of Products and Numerical Ranges, J. Math. Anal. and Appl. 17 (1967) 214-220.

[17] B. Yousefi and S. Jahedi, Composition operators on Banach spaces of formal power series, Boll. Unione Math. Ital. Sez. B Artic. Ric. Mat. (8) 6 (2003), no. 2, 481-487. 
[18] N. Zorboska, Composition operators on $S_{\alpha}$ spaces, Indiana Univ. Math. J. 39 (1990), 847-857.

Mohammad Taghi Heydari,

Department of Mathematics,

College of Sciences,

Yasouj University,

Yasouj 75914, Iran.

Email: heydari@yu.ac.ir

and

Department of Mathematics,

College of Sciences,

Higher Education Center of Eghlid,

Eghlid, Iran.

Email: heydari@eghlid.ac.ir 